\title{
Review of: "Is SARS-CoV-2 Spike glycoprotein impairing macrophage polarization via $\alpha 7$-nicotinic acetylcholine receptors?"
}

\author{
Milad Shirvaliloo ${ }^{1}$ \\ 1 Tabriz University of Medical Sciences
}

Potential competing interests: The author(s) declared that no potential competing interests exist.

It is a pleasure to see that the manuscript entitled "Is SARS-CoV-2 Spike glycoprotein impairing macrophage polarization via $\boldsymbol{\alpha} 7$-nicotinic acetylcholine receptors?" has been amended in a way to provide a better readability, while maximizing the effective delivery of its findings. Significant improvements have been made to the manuscript in comparison to the previous iteration, which is commendable. In its entirety, the paper is now giving a better impression in terms of semantic coherency and technical consistency. In contrast to the previous review, I am going to adopt a dichotomous approach to the appraisal of the present manuscript, discussing the improvable aspects in two primary sections, namely, major and minor comments.

\section{Major Comments}

1. The present manuscript is a commentary/opinion piece, and thus, inclusion of an abstract is not suggested. In fact, in the case of commentaries, abstracts are a redundancy. Nonetheless, if the composing system mandates inclusion of an abstract, which appears to be the case, the abstract should be written in as few words as possible. It should be noted that statements such as "described above" are not acceptable in an abstract, since this is not a discussion.

2. In the first paragraph of the introduction stating "Macrophages are an attractive target...", the agent to which macrophages are attractive targets should be clarified, since it is unclear whether the manuscript is referring to SARSCoV-2 or something else.

3. The terms "eicosanoid storm" and "cytokine storm" are mentioned at the end of the first paragraph in introduction, however, no further details are provided to explain each type of storm and their potential differences with each other. Particularly, the term "eicosanoid" should be further discussed.

4. The last two paragraphs in the introduction are presented in an inappropriate order. It is advised to first discuss the structure and function of $\alpha 7-\mathrm{nAChR}$, and its relation to the nervous system, and then proceed with explaining the "inflammatory reflex" in further detail; as no information is provided regarding the distribution and dynamics of different types of macrophages in the spleen.

5. The first paragraph in the section "the misdirection", biosynthesis of the furin cleavage site in the spike protein is only vaguely mentioned. The exact relationship between the S1 and S2 subunit, the cleavage site and the biosynthesis of the furin cleavage is not clear. 
6. The same paragraph also contains colloquialisms such as "a few tricks up its sleeve" which should be avoided.

7. In the second paragraph, succeeding the one mentioned above, the manuscript states "...and therefore must have a function". "Must" is not an appropriate modal verb, since it strictly excludes any other potential possibilities.

8. Conclusions are usually given in a single paragraph, briefing the key points of the manuscript. The conclusion section in this manuscript is too long. Certain parts of the conclusion, particularly the second paragraph that notes the evolution of the virus and adoption of mRNA vaccines can be expanded and included in a separate section as part of the discussion.

\section{Minor Comments}

1. The first paragraph in the section "the misdirection" includes two unnecessary question, e.g., "So why did that happen?", which can be restated in declarative sentences.

2. In the last sentence of the same section, there seems to be an error in the very last part "...that is also encoded to this cholinergic epitope"; which incorrectly implies the encoding of an antibody to a receptor.

I would like to thank the authors for their consideration. I hope that the present review will help to further improve the quality of this manuscript. 